\title{
Contemporary Hormonal Contraception and the Risk of Breast Cancer
}

\author{
Lina S. Mørch, Ph.D., Charlotte W. Skovlund, M.Sc., Philip C. Hannaford, M.D., \\ Lisa Iversen, Ph.D., Shona Fielding, Ph.D., and Øjvind Lidegaard, D.M.Sci.
}

\section{ABSTRACT}

From Rigshospitalet, the Juliane Marie Center, Department of Gynecology, University of Copenhagen, Copenhagen (L.S.M., C.W.S., Ø.L.); and Academic Primary Care (P.C.H., L.I.) and Medical Statistics (S.F.), Institute of Applied Health Sciences, University of Aberdeen, Aberdeen, United Kingdom. Address reprint requests to Dr. Mørch or Dr. Lidegaard at Rigshospitalet, Department of Gynecology, University of Copenhagen, Blegdamsvej 9, DK-2100 Copenhagen, Denmark, or at linamorch@ yahoo.dk or oejvind.lidegaard@regionh.dk.

N Engl J Med 2017;377:2228-39. DOI: 10.1056/NEJMoal700732

Copyright ( 2017 Massachusetts Medical Society.

\section{BACKGROUND}

Little is known about whether contemporary hormonal contraception is associated with an increased risk of breast cancer.

\section{METHODS}

We assessed associations between the use of hormonal contraception and the risk of invasive breast cancer in a nationwide prospective cohort study involving all women in Denmark between 15 and 49 years of age who had not had cancer or venous thromboembolism and who had not received treatment for infertility. Nationwide registries provided individually updated information about the use of hormonal contraception, breast-cancer diagnoses, and potential confounders.

\section{RESULTS}

Among 1.8 million women who were followed on average for 10.9 years (a total of 19.6 million person-years), 11,517 cases of breast cancer occurred. As compared with women who had never used hormonal contraception, the relative risk of breast cancer among all current and recent users of hormonal contraception was 1.20 (95\% confidence interval [CI], 1.14 to 1.26). This risk increased from 1.09 ( $95 \%$ CI, 0.96 to 1.23) with less than 1 year of use to 1.38 (95\% CI, 1.26 to 1.51) with more than 10 years of use $(\mathrm{P}=0.002)$. After discontinuation of hormonal contraception, the risk of breast cancer was still higher among the women who had used hormonal contraceptives for 5 years or more than among women who had not used hormonal contraceptives. Risk estimates associated with current or recent use of various oral combination (estrogen-progestin) contraceptives varied between 1.0 and 1.6. Women who currently or recently used the progestin-only intrauterine system also had a higher risk of breast cancer than women who had never used hormonal contraceptives (relative risk, 1.21; 95\% CI, 1.11 to 1.33). The overall absolute increase in breast cancers diagnosed among current and recent users of any hormonal contraceptive was 13 (95\% CI, 10 to 16) per 100,000 personyears, or approximately 1 extra breast cancer for every 7690 women using hormonal contraception for 1 year.

\section{CONCLUSIONS}

The risk of breast cancer was higher among women who currently or recently used contemporary hormonal contraceptives than among women who had never used hormonal contraceptives, and this risk increased with longer durations of use; however, absolute increases in risk were small. (Funded by the Novo Nordisk Foundation.) 
N ESTIMATED 140 MILLION WOMEN worldwide use hormonal contraception; this number accounts for approximately $13 \%$ of women between the ages of 15 and 49 years. ${ }^{1}$ In Denmark, this percentage increased from 24\% in 1995 to $39 \%$ in 2012.,3

Estrogen promotes the development of breast cancer, the leading cancer in women worldwide, whereas the role of progestin is more complex. ${ }^{4}$ Uncertainties remain regarding the association between the use of hormonal contraception and the risk of breast cancer. Previous, mainly casecontrol, studies that showed positive associations between the use of oral contraceptives and breast-cancer risk were generally conducted when the estrogen dose in combined (estrogen-progestin) hormonal contraceptives was higher than it is today and before the availability of products with new progestins and new routes of delivery. ${ }^{5}$ New methods such as the levonorgestrel-releasing intrauterine system, contraceptive patches, vaginal rings, progestin-only implants, and injections now account for almost one third of all hormonal contraceptives used in many countries, including Denmark. ${ }^{3}$ Concern regarding the progestin content of hormonal contraceptives has arisen because studies have indicated that the addition of progestin appears to increase the risk of breast cancer among postmenopausal women who receive hormone therapy. ${ }^{6-11}$

Studies of breast-cancer risk among women who receive hormonal contraceptives show inconsistent findings - from no elevation in risk to a 20 to $30 \%$ increase in risk. ${ }^{12-29}$ Most studies have assessed women according to whether they were current, recent, or past users of oral contraceptives or whether they had ever used oral contraceptives. Few studies have examined specific combined oral contraceptives containing various progestins, ${ }^{13,16,17,25,27,29}$ particularly newer products containing desogestrel, gestodene, or drospirenone, and none have been large enough to provide robust risk estimates for specific combinations. Little is known about breast-cancer risk with the use of progestin-only contraception or nonoral hormonal contraception. Also, most of the collective evidence relates to postmenopausal women, although limited information suggests that use at a young age may confer a higher risk than initiation of use later. ${ }^{5,30,31}$ We report on a nationwide epidemiologic analysis of breast-cancer risk among women of reproductive age who were using currently available hormonal contraception.

\section{METHODS}

\section{STUDY OVERSIGHT}

The Danish Data Protection Agency and the Danish Health Data Board approved the study. In Denmark, ethics approval is not required for registry-based studies. The study was supported by the Novo Nordisk Foundation, which had no role in the study design, data analysis or interpretation, writing of the manuscript, or the decision to publish the manuscript. The first and last authors vouch for the accuracy and completeness of the data and analyses.

\section{STUDY POPULATION}

The Danish Sex Hormone Register Study, which was established to assess the influence of hormone use on the risks of cardiovascular disease and cancer, follows a national cohort of Danish women between 15 and 79 years of age. The personal identification number in the Danish Civil Registration System was used to merge data from several nationwide registries (Table S1 in the Supplementary Appendix, available with the full text of this article at NEJM.org). The study has been described in detail previously. ${ }^{32,33}$ Since data from the National Register of Medicinal Product Statistics are complete from January 1, 1995, this was the study start date.

All women living in Denmark who were between 15 and 49 years of age on January 1, 1995, and those who subsequently were 15 years of age before December 31, 2012 (a total of 1,837,297 women) were eligible unless they immigrated to Denmark after 1995. After the exclusion of women who had cancer (except nonmelanoma skin cancer) or venous thromboembolism and women who had received treatment for infertility (defined as a filled prescription for ovarianstimulation drugs before study entry), a total of $1,797,932$ women were included in the study population.

\section{HORMONAL CONTRACEPTION}

The National Register of Medicinal Product Statistics provided information on filled prescriptions (Table S2 in the Supplementary Appendix). 
Use of hormonal contraception was updated throughout the follow-up period, and the status of women changed when they discontinued or changed the type of hormonal contraception used. All durations of prescriptions were extended by up to 28 days to account for delays in initiating use after the contraceptive was purchased. ${ }^{34}$ This extension was also made if no further contraceptive was purchased. Use of hormonal contraceptives was categorized as current use or recent use (discontinuation within the previous 6 months) or previous use (discontinuation more than 6 months previously). Start of use was the date that the prescription was purchased. The levonorgestrel-releasing intrauterine system was assumed to be used for 4 years, unless the woman became pregnant or another hormonal contraceptive was prescribed before the end of the 4-year period.

\section{BREAST CANCER}

The Danish Cancer Registry was used to identify primary invasive breast cancers. Cancer diagnoses were classified according to the International Classification of Diseases, 10th Revision, code C50.

\section{CONFOUNDING FACTORS}

Information about potential confounders (specified below) was ascertained from other nationwide registries (Table S1 in the Supplementary Appendix). A bias analysis assessed potential influences from unmeasured confounders.

\section{STATISTICAL ANALYSIS}

Data were analyzed according to Poisson regression with the use of SAS software, version 9.1 (SAS Institute), to calculate incidence-rate ratios (referred to as relative risks) and 95\% confidence intervals. Five-year age bands were used as a time scale in the Poisson regression. The study population was followed until the first diagnosis of breast cancer, death, registry-recorded emigration, age of 50 years, or the end of follow-up on December 31, 2012. Data on women were censored permanently at the time of a diagnosis of cancer or venous thromboembolism or the use of treatment of infertility, and they were censored temporarily during pregnancy and for 6 months after every delivery (i.e., after a pregnancy of $>22$ weeks of gestation).

Simple adjusted models included time-dependent information on use of hormonal contracep- tion, age, and calendar year. In addition, fully adjusted models included the following: level of education, parity, the polycystic ovary syndrome, endometriosis, and family history of breast or ovarian cancer. Adjustment for body-mass index, smoking status, and age of the woman at first delivery was made when this information was recorded for parous women, and these data were assessed as complete-case analyses. Subgroup analyses were conducted (Table S3 in the Supplementary Appendix). The reference group was women who had never used hormonal contraception at a given time during follow-up.

Tests for the effects of duration were performed as likelihood ratio tests comparing risk models with no history of use, previous use, and durations of use (or time since use) with the corresponding model in which duration categories were collapsed into one category. Exploratory analyses were used to test differences between relative risks associated with various preparations with the use of chi-square tests. Bonferroni correction was made for multiple comparisons in analyses comparing specific preparations with one another. We did not adjust for multiple comparisons in other analyses.

We calculated age-standardized incidence rates per 100,000 person-years using the age distribution of the cohort as the standard. Agestandardized absolute risk differences (incidence rates $^{\text {exposed }}$-incidence rates $\left.{ }^{\text {unexposed }}\right)$ and the number needed to harm (1/[incidence rates ${ }^{\text {exposed }}$ incidence rates $\left.{ }^{\text {unexposed }}\right]$ ) were also calculated.

\section{RESULTS}

From 1995 through 2012, with a mean $( \pm S D)$ follow-up of $10.9 \pm 5.8$ years, a total of 19.6 million person-years and 11,517 incident breast cancers had accumulated in 1,797,932 women between the ages of 15 and 49 years. Before the first switch to another hormonal contraceptive, 14.0 million person-years and 9101 incident breast cancers had accumulated in the cohort. Characteristics of the study population are listed in Table 1, and in Table S4 in the Supplementary Appendix.

As compared with women who had never used hormonal contraceptives, the relative risk of breast cancer among all current or recent users of any hormonal contraception was 1.20 (95\% confidence interval [CI], 1.14 to 1.26) (Table 2). The 


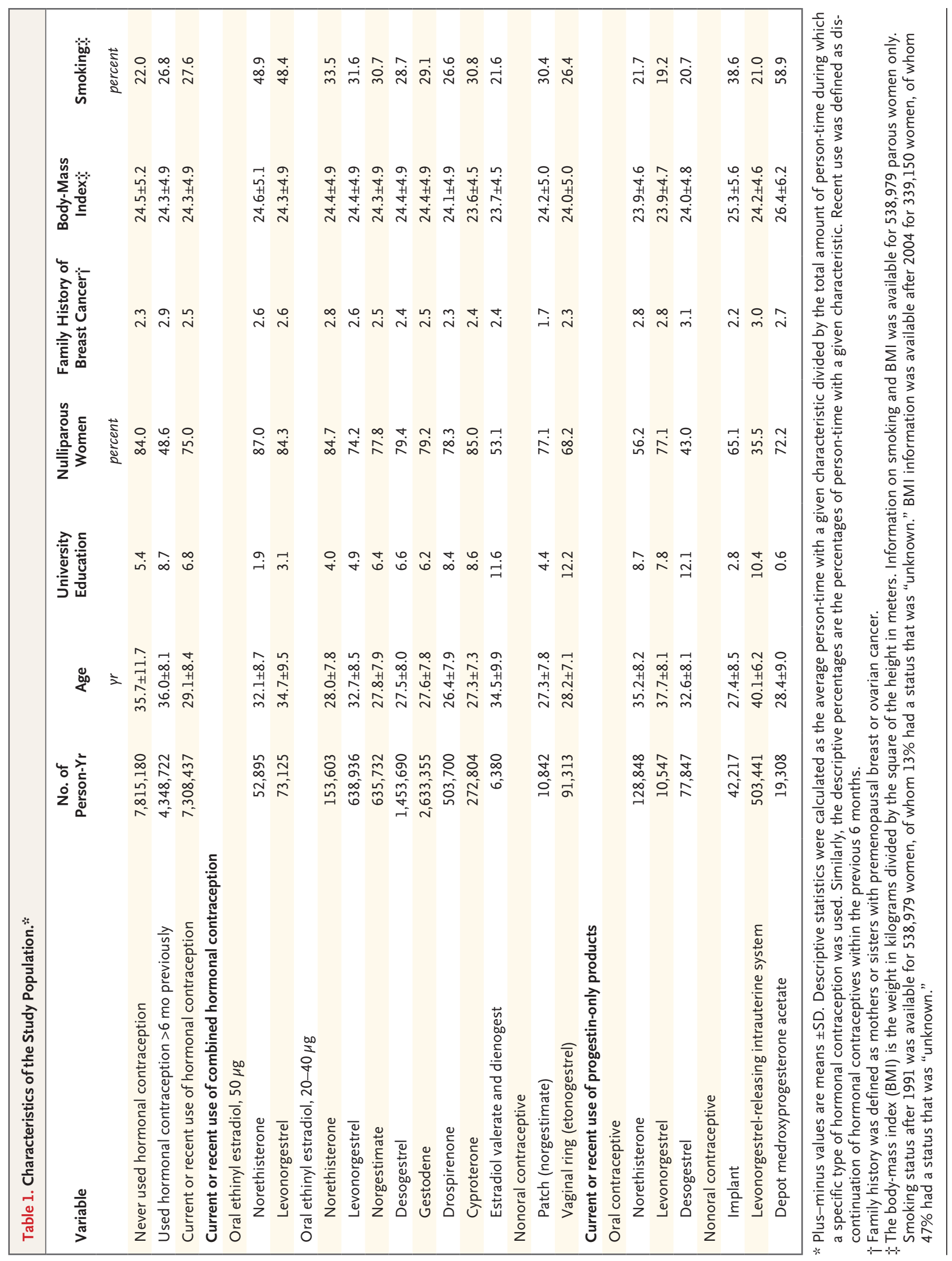

N ENGLJ MED 377;23 NEJM.ORG DECEMBER 7, 2017

The New England Journal of Medicine 


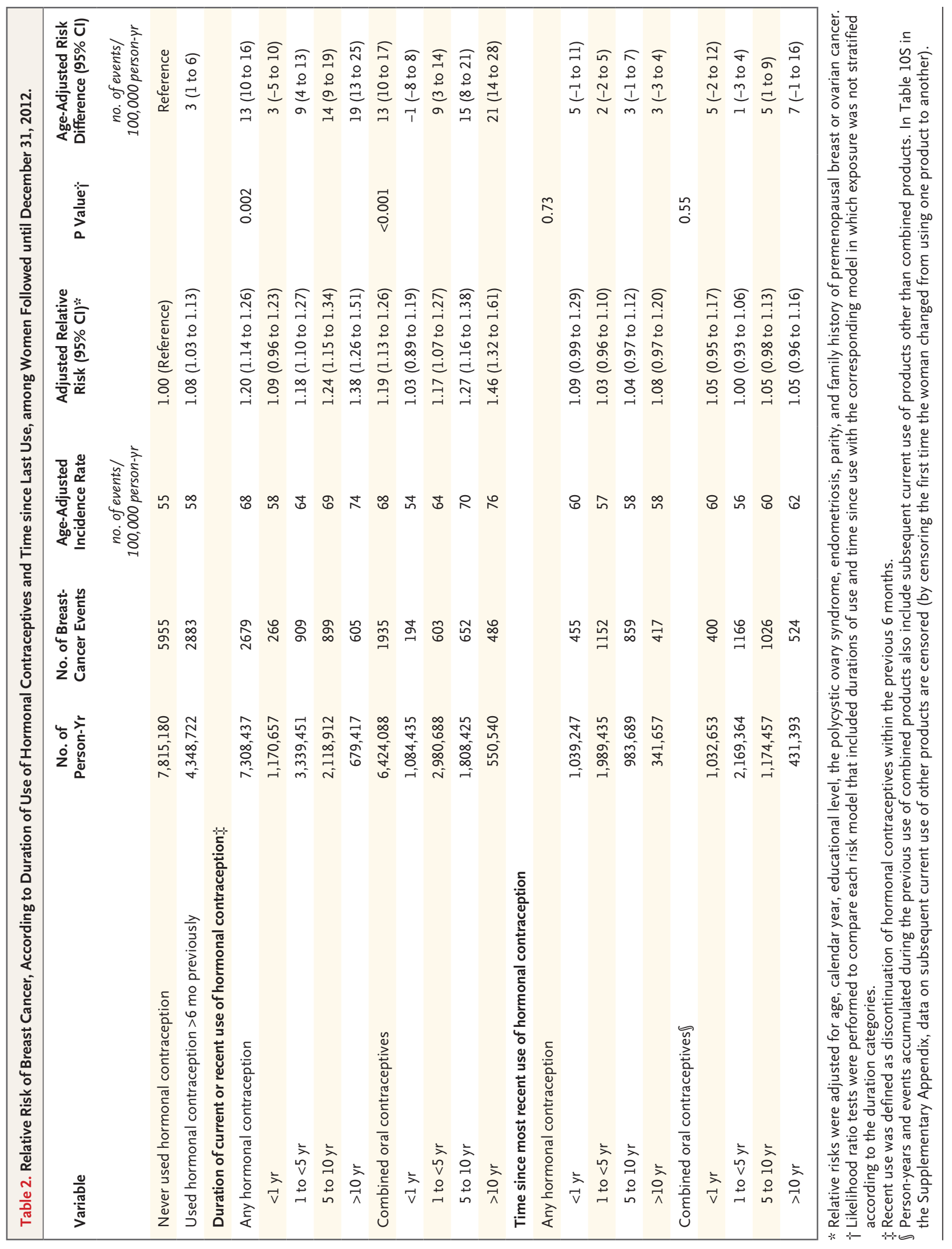


risk of breast cancer increased with the duration of use, from 1.09 (95\% CI, 0.96 to 1.23) with less than 1 year of use to 1.38 (95\% CI, 1.26 to 1.51 ) after more than 10 years of use $(\mathrm{P}=0.002)(\mathrm{Ta}-$ ble 2). As compared with women who had never used hormonal contraception, an increased risk of breast cancer was observed among woman who had previously used hormonal contraception for long periods of time (i.e., $\geq 5$ years). Among these women, the risk appeared to remain increased for at least 5 years after discontinuation (Table 3). No increased risk was found among women who had previously used hormonal contraception for less than 5 years.

There was little evidence of major differences among women who used various combined oral contraceptives (Table 4). Few significant differences were detected when various preparations were compared, and the differences were no longer significant after adjustment for multiple testing.

The relative risks of breast cancer associated with triphasic and monophasic preparations containing levonorgestrel were similar (1.21 [95\% CI, 1.04 to 1.41 ] and 1.45 [95\% CI, 1.26 to 1.67], respectively; $\mathrm{P}=0.07$ ). Risk estimates for combined pills containing norethisterone or levonorgestrel and $50 \mu \mathrm{g}$ of estrogen were similar to combinations with the same progestins combined with 20 to $40 \mu \mathrm{g}$ of estrogen (Table 4). When analyses were adjusted for the estrogen dose in each product, the overall relative risk of breast cancer associated with current or recent use was attenuated; the relative risk associated with gestodene products remained significantly elevated (Table S5 in the Supplementary Appendix). For each oral contraceptive containing 20 to $40 \mu \mathrm{g}$ of estrogen, the risk of breast cancer appeared to depend on the duration of use, although the effect of duration was significant only for products containing gestodene $(\mathrm{P}=0.003)$ (Table 5$)$.

Among women who used the levonorgestrelreleasing intrauterine system, the relative risk of breast cancer was 1.21 (95\% CI, 1.11 to 1.33 ), which did not differ significantly from the risk with products containing oral levonorgestrel alone (Table 4). There were few breast-cancer events among users of the progestin-only implant and depot medroxyprogesterone acetate.

\section{SUBGROUP AND SENSITIVITY ANALYSES}

Each subgroup analysis had less precise risk estimates than the main analyses. Even so, broadly
Table 3. Relative Risk of Breast Cancer, According to Time since Use and Duration of Use of Any Type of Hormonal Contraception, among Women Followed until December 31, 2012.*

\begin{tabular}{|lccc|}
\hline $\begin{array}{c}\text { Duration of Use } \\
\text { of Hormonal } \\
\text { Contraceptive }\end{array}$ & \multicolumn{3}{c}{ Relative Risk of Breast Cancer (95\% Cl) } \\
& $\begin{array}{c}<\text { Yr since Recent } \\
\text { Use }\end{array}$ & $\begin{array}{c}1 \text { to }<5 \text { Yr since } \\
\text { Recent Use }\end{array}$ & $\begin{array}{c}5 \text { to } 10 \text { Yr since } \\
\text { Recent Use }\end{array}$ \\
& $0.96(0.78-1.19)$ & $0.96(0.85-1.09)$ & $1.01(0.88-1.15)$ \\
$<1 \mathrm{yr}$ & $1.04(0.88-1.23)$ & $1.06(0.96-1.18)$ & $1.07(0.94-1.20)$ \\
1 to $<5 \mathrm{yr}$ & $1.33(1.11-1.59)$ & $1.16(1.02-1.33)$ & $1.30(1.06-1.58)$ \\
5 to $10 \mathrm{yr}$ & $1.52(1.17-1.98)$ & $1.16(0.89-1.49)$ & $\mathrm{NA} \uparrow$ \\
$>10 \mathrm{yr}$ & & &
\end{tabular}

* Use of hormonal contraceptives included any type of hormonal contraception. Rate ratios and $95 \%$ confidence intervals were adjusted for age, calendar year, level of education, the polycystic ovary syndrome, endometriosis, parity, and family history of premenopausal breast or ovarian cancer. The reference group was women who had never used hormonal contraception. Recent was defined as discontinuation of hormonal contraception within the previous 6 months. NA denotes not applicable.

$\uparrow$ The number of events was too small to determine risk estimates.

similar risk patterns were observed among women who had used hormonal contraceptives for at least 5 years before inclusion in the analysis (Table S6 in the Supplementary Appendix). Risk estimates among women who were younger than 35 years of age showed limited evidence of major differences among the products, apart from a suggestion of larger risk estimates for levonorgestrel products (Table S7 in the Supplementary Appendix). Results among nulliparous women were generally similar to those of the main analyses (Table S8 in the Supplementary Appendix). There was a suggestion that initiation of use of hormonal contraceptives before 20 years of age may be associated with enhanced risks of breast cancer, especially with long durations of use, although the risk estimates were imprecise (Table S9 in the Supplementary Appendix). Results among women who were followed until the first change from one type of hormonal contraception to another were similar to those in the main analyses. Details are provided in Table S10 through S12 in the Supplementary Appendix.

Sensitivity analyses with adjustment for age according to 1-year age groups did not materially change the main estimates. Simple and fully adjusted results were similar. Additional adjustment for body-mass index, smoking, and age at first birth in parous women did not materially change estimates. A quantitative bias analysis 





showed that a hypothetical unmeasured confounder would need to have a $50 \%$ prevalence in the population, increase the risk of breast cancer by a factor of 3 , and increase the chance of using hormonal contraception by 2.5 times in order to eliminate the observed relative risk with current or recent use of hormonal contraception (Fig. S1 in the Supplementary Appendix).

The age-standardized incidence rates for breast cancer and absolute risk differences per 100,000 person-years are shown in Tables 2, 4, and 5. The difference in the risk of breast cancer between women who had never used hormonal contraception and current and recent users of any type of hormonal contraception was 13 (95\% CI, 10 to 16) per 100,000 person-years; approximately one extra breast cancer was diagnosed for every 7690 women using hormonal contraception for 1 year.

\section{I SCUSSION}

In this prospective cohort study, the current or recent use of hormonal contraception was associated with a higher risk of breast cancer than the risk among women who had never used hormonal contraceptives, with little evidence of major differences between specific combined oral contraceptives. The risk increased with the duration of use.

The collaborative reanalysis of data from individual women ${ }^{5}$ showed that among the women who were currently using combined oral contraceptives, the relative risk of breast cancer was 1.24 (95\% CI, 1.15 to 1.33), which is close to our estimate of 1.19 (95\% CI, 1.13 to 1.26) for all combined oral products. In line with our data, other studies have not shown consistent differences among women who used older combined oral contraceptives (from the 1970s and 1980s) that had a different progestin content. ${ }^{13,15,16,18,25,29}$

Our data showed a higher risk of breast cancer with a longer duration of use of combined products with various progestins, although the effect of duration was only significant for the largest product group containing gestodene. A duration-of-use relationship has not been shown in most studies assessing long-term contraceptive use, ${ }^{5,21}$ possibly because many included a large proportion of postmenopausal women whose past use of oral contraceptives before menopause was unlikely to influence the breast-cancer risk estimates greatly. ${ }^{5,13,27}$ Studies that have stratified data according to menopausal status have also shown a higher risk of breast cancer among premenopausal woman who have used hormonal contraceptives for long periods of time than among those who have used them for short periods of time. ${ }^{22,24,25}$

Our results suggest the rapid disappearance of excess risk of breast cancer after discontinuation of use among women who have used hormonal contraceptives for short periods, whereas the risk among women who have used these contraceptives for longer periods may persist for at least 5 years after discontinuation. In the collaborative reanalysis, the slightly higher risk among former users of combined oral contraceptives disappeared within 5 years. ${ }^{5}$ Other studies have shown no evidence of persisting risk several years after the discontinuation of oral contraception. ${ }^{22,24,25}$ Study differences may be due to variations in the mean duration of use. Since our data mostly comprised women who used hormonal contraception for less than 5 years, our overall results indicated only a slightly higher risk of breast cancer among past users than among women who had never used hormonal contraception.

Few studies have assessed progestin-only contraception and breast-cancer risk. In a cohort of 93,843 women who used the levonorgestrelreleasing intrauterine system, a relative risk of breast cancer of 1.19 (95\% CI, 1.13 to 1.25) was found, as compared with the general incidence rate among Finnish women younger than 55 years of age $\mathrm{e}^{35}$; we found a similar risk estimate. The absence of an association between the duration of use of the levonorgestrel-releasing intrauterine system and breast-cancer risk might be explained by the system providing a decreasing dose of progestin released according to the time since insertion. Studies suggest a considerable systemic uptake of levonorgestrel in women who use the intrauterine system, with plasma concentrations in some women that are similar to those in women who use levonorgestrel-only pills..$^{36-38}$

Our nationwide prospective study involving 1.8 million Danish women who were followed for almost 11 years adds substantively to the sparse evidence base about contemporary hormonal contraception. The linkage of cancer and pharmacy records allowed us to incorporate timevarying exposure information about changes in 









N ENGLJ MED 377;23 NEJM.ORG DECEMBER 7, 2017

The New England Journal of Medicine 
contraceptives used and discontinuation of use. The large number of events allowed for assessment of recently marketed combined preparations, various durations of use, progestin-only products, and various routes of administration, including the intrauterine system.

This study had some limitations. We were not able to adjust for age at menarche, breast-feeding, alcohol consumption, or physical activity, and we had information on body-mass index only for parous women. Although some of these variables may be correlated with both breast-cancer risk and hormonal contraception, they would be expected to continue to influence a woman's risk after discontinuation of hormonal contraceptives; in contrast, we found that risks among women who used hormonal contraception for less than 5 years rapidly decreased after discontinuation of hormonal contraception.

Furthermore, we would not expect differences in the levels of these potential confounding factors with various durations of hormonal contraceptive use. Information about some of the potential confounders that we included in our models was not available for the oldest women, because the Danish National Health Register started in 1976. Analyses that were restricted to women younger than 35 years of age showed larger risk estimates for combined products with levonorgestrel and for the levonorgestrel-releasing intrauterine system, suggesting that missing information about potential confounders in older women is unlikely to have led to overestimation of overall risk estimates for these products. Residual confounding is also possible; for example, information about the polycystic ovary syndrome and endometriosis was available only for women who had been hospitalized with these conditions, and we adjusted only for a family history of premenopausal breast or ovarian cancer. Women with any family history of breast cancer might be less likely to use hormonal contraception, which might underestimate the risk estimate. ${ }^{4,6-11}$ Quantitative bias analysis, however, suggests that to explain the main finding, a confounder would need to be highly prevalent in the population $(50 \%)$ and strongly associated with breast cancer (by a factor of 3) and use of hormonal contraception (an odds ratio of 2.5) (Fig. S1 in the Supplementary Appendix); such an unknown confounder appears to be unlikely. Information was not available about exposure to hormonal contraceptives before study entry. Some women who did not purchase a hormonal contraceptive during the study period (and so were classified as never having used hormonal contraception) may have used hormonal contraception beforehand. Such misclassification (left censor bias) would underestimate breast-cancer risk among women who used hormonal contraceptives. Analyses that were restricted to women with at least 5 years of contraceptive history before inclusion in the analysis generally produced results that were similar to those of the main analysis. Some women might have stopped taking hormonal contraception even though their prescription length indicated that they were still users; such misclassification would also be expected to lead to underestimates of the risk of breast cancer among women who use hormonal contraception.

If women who were currently using hormonal contraception were screened for breast cancer more often than those who were not, we would expect to see a decreased breast-cancer risk among former users because of a reduced detection rate after the more intensive screening while the women were current users. This was not apparent in our data. Finally, we did not adjust for multiple statistical testing in the main analyses, and this might explain some of the associations observed. However, the findings were consistent across many analyses including various types of hormonal contraception.

The estimated number of additional breast cancers that were associated with hormonal contraception did not include extra cases diagnosed after the discontinuation of long-term use. Even so, the estimated number of additional breast cancers among premenopausal women that were attributable to hormonal contraception is likely to be low. This risk should be weighed against important benefits of hormonal contraceptives such as good contraceptive efficacy and reduced risks of ovarian, endometrial, and perhaps colorectal cancer (at least for combined oral contraceptives that were commonly used in the 1970s and 1980s). ${ }^{39}$

Supported by a grant (11645) from the Novo Nordisk Foundation.

Drs. Mфrch and Skovlund became employed by Novo Nordisk Foundation after the manuscript was accepted for publication. No other potential conflict of interest relevant to this article was reported.

Disclosure forms provided by the authors are available with the full text of this article at NEJM.org. 


\section{REFERENCES}

1. World contraceptive patterns 2013. New York: United Nations, Department of Economic and Social Affairs (http://www .un.org/en/development/desa/population/ publications/family/contraceptive-wallchart -2013.shtml).

2. Wilson NM, Laursen M, Lidegaard $\varnothing$. Oral contraception in Denmark 1998-2010. Acta Obstet Gynecol Scand 2012;91:810-5 3. Lindh I, Skjeldestad FE, GemzellDanielsson K, et al. Contraceptive use in the Nordic countries. Acta Obstet Gynecol Scand 2017;96:19-28.

4. Pasqualini JR. Progestins and breast cancer. Gynecol Endocrinol 2007;23. Suppl 1:32-41.

5. Collaborative Group on Hormonal Factors in Breast Cancer. Breast cancer and hormonal contraceptives: collaborative reanalysis of individual data on 53297 women with breast cancer and 100239 women without breast cancer from 54 epidemiological studies. Lancet 1996;347:1713-27.

6. Pike MC, Ross RK. Progestins and menopause: epidemiological studies of risks of endometrial and breast cancer. Steroids 2000;65:659-64.

7. Ross RK, Paganini-Hill A, Wan PC Pike MC. Effect of hormone replacement therapy on breast cancer risk: estrogen versus estrogen plus progestin. J Natl Cancer Inst 2000;92:328-32.

8. Schairer C, Lubin J, Troisi R, Sturgeon S, Brinton L, Hoover R. Menopausal estrogen and estrogen-progestin replacement therapy and breast cancer risk. JAMA 2000 283:485-91.

9. Colditz GA, Rosner B. Cumulative risk of breast cancer to age 70 years according to risk factor status: data from the Nurses' Health Study. Am J Epidemiol 2000;152 950-64.

10. Chlebowski RT, Hendrix SL, Langer $\mathrm{RD}$, et al. Influence of estrogen plus progestin on breast cancer and mammography in healthy postmenopausal women: the Women's Health Initiative Randomized Trial. JAMA 2003;289:3243-53.

11. Chlebowski RT, Kuller LH, Prentice $\mathrm{RL}$, et al. Breast cancer after use of estrogen plus progestin in postmenopausal women. N Engl J Med 2009;360:573-87.

12. Van Hoften C, Burger $\mathrm{H}$, Peeter PHM, Grobbee DE, Van Noord PAH, Leufkens HG. Long-term oral contraceptive use increases breast cancer risk in women over 55 years of age: the DOM cohort. Int J Cancer 2000;87:591-4.

13. Marchbanks PA, McDonald JA, Wilson $\mathrm{HG}$, et al. Oral contraceptives and the risk of breast cancer. N Engl J Med 2002;346: 2025-32.

14. Kumle M, Weiderpass E, Braaten $T$, Persson I, Adami HO, Lund E. Use of oral contraceptives and breast cancer risk: the Norwegian-Swedish Women's Lifestyle and
Health Cohort Study. Cancer Epidemiol Biomarkers Prev 2002;11:1375-81.

15. Althuis MD, Brogan DD, Coates RJ, et al. Breast cancers among very young premenopausal women (United States). Cancer Causes Control 2003;14:151-60.

16. Althuis MD, Brogan DR, Coates RJ, et al. Hormonal content and potency of oral contraceptives and breast cancer risk among young women. Br J Cancer 2003; 88:50-7.

17. Dumeaux V, Alsaker E, Lund E. Breast cancer and specific types of oral contraceptives: a large Norwegian cohort study. Int J Cancer 2003;105:844-50.

18. Dumeaux V, Fournier A, Lund E, Clavel-Chapelon F. Previous oral contraceptive use and breast cancer risk according to hormone replacement therapy use among postmenopausal women. Cancer Causes Control 2005;16:537-44.

19. Lee SY, Kim MT, Kim SW, Song MS, Yoon SJ. Effect of lifetime lactation on breast cancer risk: a Korean women's cohort study. Int J Cancer 2003;105:390-3.

20. Wu MH, Chou YC, Yu JC, et al. Hormonal and body-size factors in relation to breast cancer risk: a prospective study of 11,889 women in a low-incidence area. Ann Epidemiol 2006;16:223-9.

21. Hannaford PC, Selvaraj S, Elliott AM, Angus V, Iversen L, Lee AJ. Cancer risk among users of oral contraceptives: cohort data from the Royal College of General Practitioner's Oral Contraception Study. BMJ 2007;335:651.

22. Shantakumar S, Terry MB, Paykin A, et al. Age and menopausal effects of hormonal birth control and hormone replacement therapy in relation to breast cancer risk. Am J Epidemiol 2007;165:1187-98. 23. Dorjgochoo T, Shu XO, Li HL, et al. Use of oral contraceptives, intrauterine devices and tubal sterilization and cancer risk in a large prospective study, from 1996 to 2006. Int J Cancer 2009;124:2442-9.

24. Rosenberg L, Zhang Y, Coogan PF, Strom BL, Palmer JR. A case-control study of oral contraceptive use and incident breast cancer. Am J Epidemiol 2009;169: 473-9.

25. Hunter DJ, Colditz GA, Hankinson SE, et al. Oral contraceptive use and breast cancer: a prospective study of young women. Cancer Epidemiol Biomarkers Prev 2010;19:2496-502.

26. Kawai M, Minami Y, Kuriyama S, et al. Reproductive factors, exogenous female hormone use and breast cancer risk in Japanese: the Miyagi Cohort Study. Cancer Causes Control 2010;21:135-45.

27. Marchbanks PA, Curtis KM, Mandel MG, et al. Oral contraceptive formulation and risk of breast cancer. Contraception 2012;85:342-50.

28. Vessey M, Yeates D. Oral contracep- tive use and cancer: final report from the Oxford-Family Planning Association contraceptive study. Contraception 2013;88: 678-83.

29. Beaber EF, Buist DSM, Barlow WE, Malone KE, Reed SD, Li CI. Recent oral contraceptive use by formulation and breast cancer risk among women 20 to 49 years of age. Cancer Res 2014;74:4078-89. 30. Jernström $H$, Loman $N$, Johannsson OT, Borg A, Olsson H. Impact of teenage oral contraceptive use in a populationbased series of early-onset breast cancer cases who have undergone BRCA mutation testing. Eur J Cancer 2005;41:2312-20.

31. Kotsopoulos J, Lubinski J, Moller P, et al. Timing of oral contraceptive use and the risk of breast cancer in BRCA1 mutation carriers. Breast Cancer Res Treat 2014; 143:579-86

32. Mфrch LS, Lфkkegaard E, Andreasen $\mathrm{AH}$, Krüger-Kjaer S, Lidegaard O. Hormone therapy and ovarian cancer. JAMA 2009;302:298-305.

33. Lidegaard $\phi$, L $\phi$ kkegaard E, Jensen A, Wessel Skovlund C, Keiding N. Thrombotic stroke and myocardial infarction with hormonal contraception. N Engl J Med 2012;366:2257-66.

34. Nielsen LH, Lфkkegaard E, Andreasen $\mathrm{AH}$, Keiding N. Using prescription registries to define continuous drug use: how to fill gaps between prescriptions. Pharmacoepidemiol Drug Saf 2008;17:384-8.

35. Soini T, Hurskainen R, Grénman S, Mäenpää J, Paavonen J, Pukkala E. Cancer risk in women using the levonorgestrelreleasing intrauterine system in Finland. Obstet Gynecol 2014;124:292-9.

36. Lockhat FB, Emembolu JE, Konje JC. Serum and peritoneal fluid levels of levonorgestrel in women with endometriosis who were treated with an intrauterine contraceptive device containing levonorgestrel. Fertil Steril 2005;83:398-404.

37. Raudaskoski T, Tapanainen J, Tomás E, et al. Intrauterine 10 microg and 20 microg levonorgestrel systems in postmenopausal women receiving oral oestrogen replacement therapy: clinical, endometrial and metabolic response. BJOG 2002;109:13644.

38. Haimov-Kochman $\mathrm{R}$, Amsalem $\mathrm{H}$, Adoni A, Lavy Y, Spitz IM. Management of a perforated levonorgestrel-medicated intrauterine device - a pharmacokinetic study: case report. Hum Reprod 2003;18: 1231-3.

39. Iversen L, Sivasubramaniam S, Lee AJ, Fielding S, Hannaford PC. Lifetime cancer risk and combined oral contraceptives: the Royal College of General Practitioners' Oral Contraception Study. Am J Obstet Gynecol 2017;216(6):580.e1-580.e9.

Copyright (c) 2017 Massachusetts Medical Society. 